\title{
Matching in the presence of don't cares and redundant sequential elements for sequential equivalence checking
}

\author{
Solaiman Rahim \\ rahim@synplicity.com \\ LIRMM - Synplicity \\ Montpellier, FR
}

\author{
Bruno Rouzeyre \\ rouzeyre@lirmm.fr \\ LIRMM \\ Montpellier, FR
}

\author{
Lionel Torres \\ torres@lirmm.fr jerome@synplicity.com \\ LIRMM \\ Montpellier, FR
}

\begin{abstract}
:
Full sequential equivalence checking by state space traversal has been shown to be unpractical for large designs [10]. To address state space explosion new approaches have been proposed that exploit structural characteristics of a design and make use of multiple analysis engines (e.g. BDDs, Simulation, SAT) to transform the sequential equivalence checking problem into a combinational equivalence checking problem [1][8]. While these approaches, based on induction techniques [1][2][3], have been successful in general, they are not able to reach proof of equivalence in presence of complex transformations between the reference design and its implementation. One of these transformations is redundant Flip-Flops (FFs) removal. FFs may be removed by redundancy removal, or don't care optimization techniques applied by synthesis tools. Consequently, some FFs in the reference design may have no equivalent FFs in the implementation net-list. Latest researches in this area have proposed specific solutions for particular cases. In [5], matching in the presence of redundant constant input FFs has been addressed and in [11] identification of sequential redundancy is performed. This paper presents an indepth study of some possible causes of unmatched FFs due to redundancy removal, and proposes a generic approach to achieve prove of equivalence in presence of redundant FFs. Our approach is independent from specific synthesis transformations. It is able to achieve matching in presence of complex redundancies, and is able to perform formal equivalence checking in presence of don't cares. The experimental results show a significant improvement in the matching rates of FFs when compared to industrial equivalence checking tools. This higher matching is directly translated to a higher success rate in proving equivalency.
\end{abstract}

\section{Introduction}

Generic equivalence checking methods of two sequential circuits require a state space traversal of the product machine. This method has the capacity to handle sequential optimizations (such as retiming, pruning, state machine re-encoding....), which are performed during synthesis. However, due to computational complexity, those methods cannot be applied to large circuits. Other approaches have been investigated which try to map the sequential equivalence problem of circuits into a combinational equivalence checking problem [8]. They rely on identifying some potential equivalent FFs or nets in the two circuits under verification through a matching/mapping step, and checking the equivalence of those FFs and nets by using combinational verification. The powerful existing matching methods [1][2][3] are functional based method using induction proof. They are applied to try to handle some sequential optimizations done during synthesis as merge, replication, sequential redundancy removal and retiming. These methods consist to find functional equivalent FFs in the two designs. But they cannot handle efficiently all type of sequential optimizations done during synthesis, particularly the optimizations which removed sequential elements such as redundancy removal or don't care optimizations (only method for constant input FFs was proposed in [5] and [6]). Furthermore, they do not handle unspecified values (which is called don't care) derived from the circuit (one method was proposed in [2] which consists to do the matching for each possible value of the don't care, but can not be applied for circuits with large number of don't care). In this paper, optimizations that remove sequential elements are presented. The problems, they may introduce for the existing matching methods, are illustrated on a set of circuits with redundant FFs. Then a synthesis independent method to complete the existing matching to handle those optimizations is proposed. We demonstrate that it completes the existing matching method efficiency. In section 6, a set of industrial circuits is used to compare our algorithm with some industrial tools

\section{Preliminaries}

Here we introduce the notion of redundant FFs and don't care functions which are used in the following sections.

\subsection{Redundant FFs}

Definition 1: A FF $\mathrm{R}$ of a circuit $\mathrm{C}$ is called redundant $F F$, if the observable input-output behavior of $\mathrm{C}$ is invariant after removing $\mathrm{R}$ from the circuit.

Definition 2: A constant input $F F$ is an FF with a constant input function ( 0 or 1$)$ at any clock cycle. 


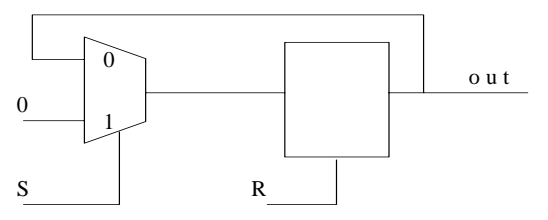

Figure 1: Example of stuck at $0 \mathrm{FF}$

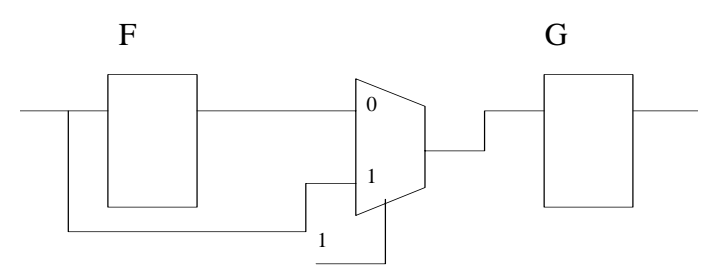

Figure 2: $\mathrm{F}$ is a non-observable $\mathrm{FF}$

Definition 3: If $\mathrm{F}(\mathrm{t})$ is the input function of an $\mathrm{FF} R$ and $\mathrm{v}$ its output variable, $\mathrm{R}$ is stuck at $O$ (resp 1) (An example is given in figure 1) iff:

1) $\exists \mathrm{t} \mid \forall \mathrm{T}>=\mathrm{t}, \mathrm{F}(\mathrm{t})=0($ resp 1$)$

2) $F(t)$ is a function of $v$.

Definition 4: An FF $\mathrm{R}$ of a circuit $\mathrm{C}$ is called a nonobservable $F F$, if the observable output behavior of $\mathrm{C}$ is independent of the value of $\mathrm{R}$, for all possible states and all possible input combinations of C. An example is given in Figure 2.

Constant input FF, stuck at FF and non-observable FF are all redundant FFs and can be removed by the synthesis tools.

\subsection{Don't care definitions}

Definition 5: A don't care variable (noted dc-var) is a Boolean function which can be substituted by any Boolean function, in particularly by 0 or 1 . Figure 3 described the use of don't care variables to express the output function of a multiple driver net.

\begin{tabular}{|c|c|c|c|}
\hline$\underline{E} 1$ & \multirow{8}{*}{ O u t } & (V1,E1,V2,E2) & Out \\
\hline $\mathrm{V} 1$ & & $(0,0,0,0)$ & Dc1 \\
\hline \multirow{6}{*}{$\begin{array}{l}\mathrm{E} 2 \\
\underline{\mathrm{V} 2} \\
\underline{4}\end{array}$} & & $(0,0,1,0)$ & Dc1 \\
\hline & & $(0,1,1,1)$ & Dc2 \\
\hline & & $(1,0,0,0)$ & De1 \\
\hline & & $(1,0,1,0)$ & Dc1 \\
\hline & & $(1,1,0,1)$ & Dc2 \\
\hline & & $(1,1,1,1)$ & $\mathrm{V} 1=\mathrm{V} 2=1$ \\
\hline $\begin{array}{l}\text { Figu } \\
\text { with }\end{array}$ & reuit & $\begin{array}{l}\text { Incomplete } 1 \\
\text { function Out }\end{array}$ & h Table of \\
\hline
\end{tabular}

Dc1 comes from the condition that E1 and E2 can both be inactive.

Dc2 comes from the condition that E1 and E2 can be active simultaneously and $\mathrm{v} 1 \neq \mathrm{v} 2$.
Definition 6: A don't care function (noted dc-fullfunc) is a Boolean function with only don't care variables in its support set. Note that dc-full-func is always reduced to 0 or 1 by affecting don't care variables to a constant values. (Figure 5)

Let Fct be the set of all Boolean functions.

Let $X=\{$ Dc1, Dc2 ... $\}$ be the set of dc-var of a Boolean function $\mathrm{F}$.

Definition 7: A dc-var-interpretation is a map $\mathrm{X}->\{0,1\}$ which associates a Boolean value to each dcvar.

Definition 8: A dc-func-interpretation of the function $\mathrm{F}$ is a map I: $\{0,1\}^{\wedge} \mathrm{X}->$ Fct which associates a Boolean function to each dc-var interpretation.

Note that all dc-func-interpretations of the function $\mathrm{F}$ are included to it and there are $2^{\wedge}|\mathrm{X}|$ dc-func interpretations of the function $\mathrm{F}$ possible.

Definition 9: $\mathrm{R}$ is a constant input FFs "modulo don't care variables" iff $\exists$ a dc-func-interpretation of $\mathrm{R} / \mathrm{R}=$ 0 or $\mathrm{R}=1$. (Example in Figure 4)

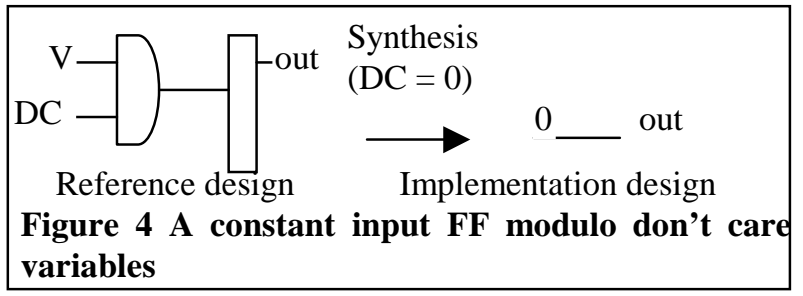

Definition 10: A zero (resp one) don't care (noted 0dc-func) is a function equal to zero (resp one) out of the don't care set, i.e $\exists$ a dc-func-interpretation of F/ F $=0($ resp 1). (Example in figure 7$)$. Notice that the example in figure 6 is a 0 -dc-func and a 1 -dc-func.

Definition 11: A function Impl is contained to a function Ref iff $\exists$ a dc-func-interpretation I of Ref such as $\mathrm{I}=\mathrm{A}$.

Theorem: A FF R is a constant input FF modulo don't care variable iff the input function of $\mathrm{R}$ (noted $\mathrm{F}$ ) is a dc-all-func, 0 -dc-func or a 1-dc-func.

If the input function of an FF R is a constant input FF modulo don't care variable, it may become a redundant FF and may be removed by the synthesis tool depending on which dc-func-interpretation of $\mathrm{R}$ is chosen. 


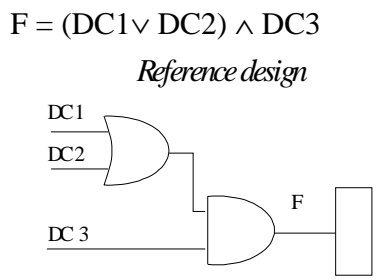

Figure 5: dc-all-func

$\mathrm{F}=(\mathrm{v} \vee \mathrm{DC} 1) \wedge \mathrm{DC} 2$

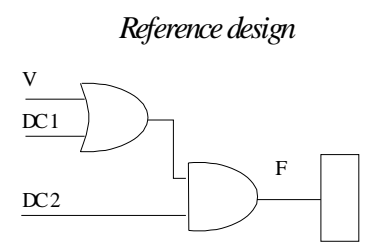

Figure 6: 0 and 1 - dcfunc

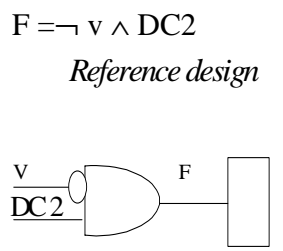

Figure 7: 0-dc-func
BDD representation

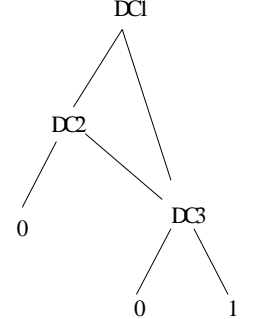

$\mathrm{BDD}$ representation

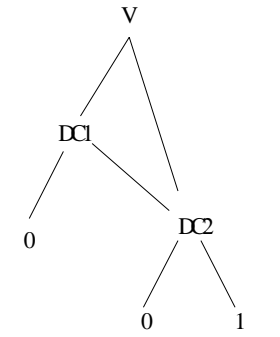

BDD representation

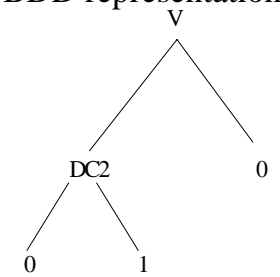

\section{Redundancy removal problems in existing matching methods $[1,2,3]$}

\subsection{Constant input FFs problems}

Let's consider the example below (Figure 8 (a),(b)). In figure $8 \mathrm{a}$, constant propagation is not performed which leads to Out $1 \neq$ Out2 (false negative). In figure $8 \mathrm{~b}$, constant propagation through FFs was performed and the proof concludes Out $1=$ Out 2 .

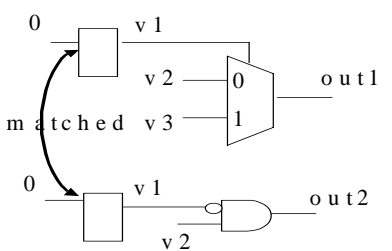

Figure8(a):Constant input

\section{FF}

Out1 $=(\mathrm{V} 2 \wedge \neg \mathrm{V} 1) \vee(\mathrm{V} 3 \wedge \mathrm{V} 1)$

Out2 $=(\mathrm{V} 2 \wedge \neg \mathrm{V} 1)$

$\Rightarrow$ Out $1 \neq$ Out $2($ False negative $)$

\subsection{Stuck at FFs problems}

The matching process identifies the redundant FFs in the reference design as unmatched FFs because there are no equivalent FFs in the implementation and its input function is not a constant. Sequential equivalence checking often considers unmatched FFs as retimed, and methods similar to [1] or [4] are applied.
The method proposed in [1] consists of finding equivalent nets. This method fails in figure 9 because there are no equivalent nets.

In [4], the method consists of injecting the input function of the unmatched FFs to its output at the next clock cycle. This method fails in figure 9 because their method of transforming the circuit with a feedback loop into a loop free circuit requires a positive unate function (otherwise an infinite loop can be created which is the case in figure 9). This is why we are proposing a method to extend the coverage in such cases.

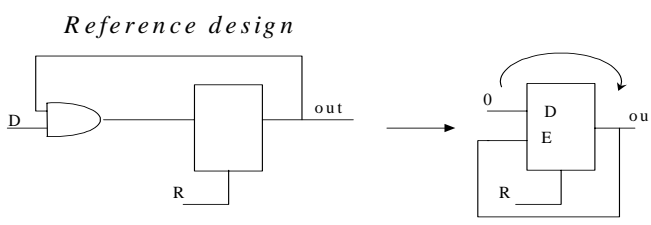

Implementation design out

Figure 9: Example of stuck at 0 FF that cannot be handled in [4]

\section{Our redundancy removal engine for matching step}

We introduce here our redundancy removal engine and the process of our engine is illustrated in Figure 10, 11, 12,13 .

\subsection{Constant input FFs}

To handle constant input FFs, the input function of some candidate FFs is computed using BDD representation. Then the constant propagation through the FFs with BDD equal to 0 and 1 is done and the FFs are removed. The candidate FFs come from a random simulation. This reduces the number of BDD computed.

\subsection{Stuck at FFs}

We propose here to extend the method proposed in [1]. Indeed, an induction technique is used here to find stuck at FFs.

Let $\mathrm{F}$ be the input function of a FF R.

- If $\mathrm{R}$ is a FF with a reset line, it can be removed if $\mathrm{F}(\mathrm{t})$ $=0 \Rightarrow \mathrm{F}(\mathrm{t}+1)=0$.

- If $\mathrm{R}$ is a FF with a set line, it can be removed if $\mathrm{F}(\mathrm{t})=$ $1=\mathrm{F}(\mathrm{t}+1)=1$.

\subsection{Non observable FFs}

The method is based on the DTPG algorithm proposed in [7] which consists of identifying redundant nodes by searching for undetectable stuck-at-fault. We apply the algorithm to the FFs instead of the circuit nodes. The 
FF is removed if no pattern is found to make the output of the FF observable.

One way to implement the DTPG algorithm is to check if the output variable of a candidate FF is present in the function of all FFs in its transitive fan out (using Bdds for example). This can be very time consuming because of the Bdds complexity. But the use of simulation techniques on the Bdds shows that the time can be considerably decreased (a average gain of $80 \%$ has been noticed)

The final flow is the following:

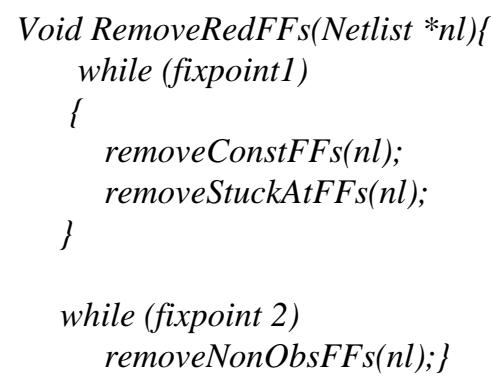

Note that our engine is a fixpoint algorithm because every time a FF is removed from the design, new FFs can become redundant. Two fixpoints is needed because removing constant input or stuck at FFs does not imply new non-observable FFs and removing non-observable FFs does not imply new constant or stuck at FFs.

The process of the algorithm is illustrated on the reference and implementation designs in Figure 10.

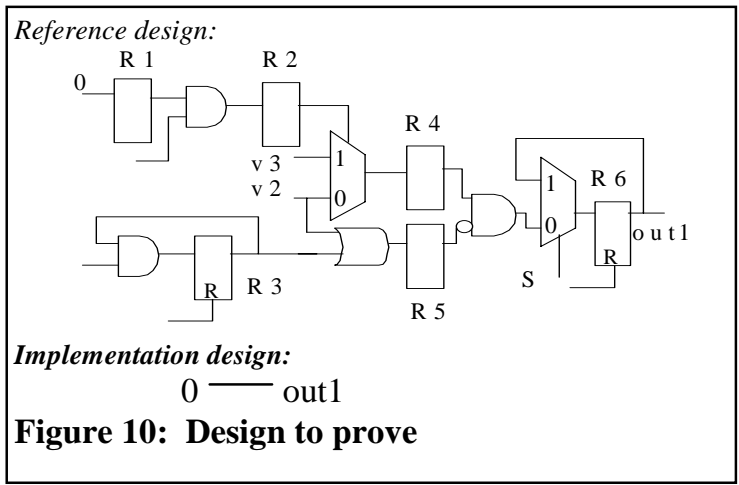

After first iteration of fixpoint 1, R1 is removed because it is a constant input $0 \mathrm{FF}, \mathrm{R} 3$ is removed because it is a stuck-at-0 FF, then the reference design is transformed into the equivalent circuit in figure 11.

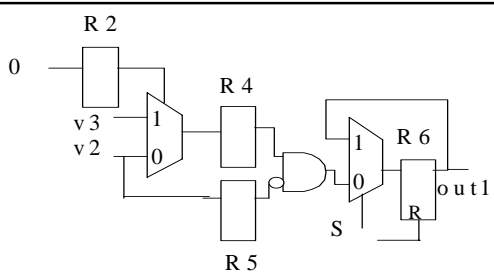

Figure 11: First iteration of fixpoint 1
After second iteration of fixpoint1, R2 is removed because it is a 0 constant input FF and the circuit in figure 11 is transformed to the equivalent circuit in Figure 12.

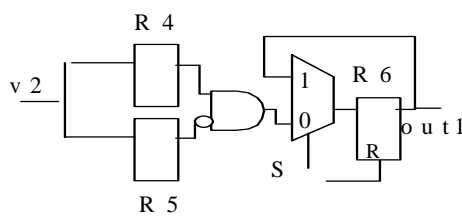

Figure 12: Second iteration of fixpoint 1

After first iteration of fixpoint1 done after the matching, R6 is removed because it became a stuck at 0 FF after that R4 and R5 have been matched together. Then the circuit in Figure 12 is transformed to the equivalent circuit in Figure 13 and can be proved easily with the implementation design

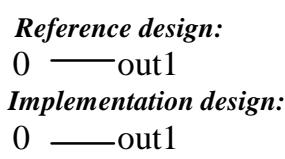

Figure 13: First iteration of fixpoint 1 done after the matching

\section{How to handle Don't care?}

Notation: In the following, a function $\mathrm{F}$ at next clock cycle (defined as the CBF function in [4]) is noted F'.

\subsection{How to handle dc-full-func}

To handle dc-full-functions, we reintroduce the don't care input function of the FFs to its output at the next clock cycle (note that a dc-full-func at time t remains a dc-full-func at time $t+1$ ). This works because dc-fullfunction is always reduced to 0 or 1 after don't care assignment, and 0 and 1 are contained to the new dcfull-func created at time $t+1$. This technique is illustrated in figure 14.

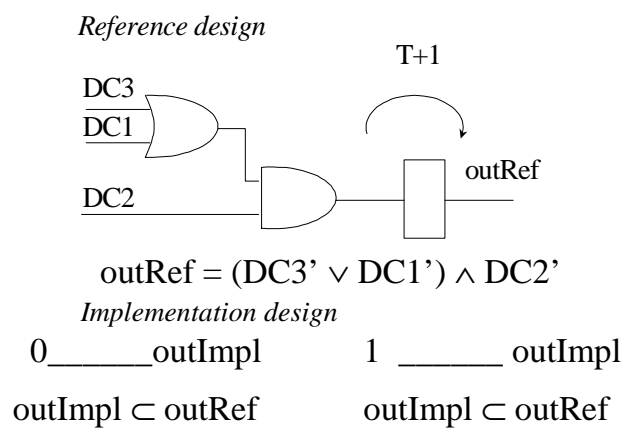

Figure 14: Different implementations of a dcfull-func 
5.2 A simulation and assertion method to handle 0-dc-func (resp 1-dc-func).

Let's consider Figure 15 and 16 that are the results of a different interpretation of the don't care variables from the same reference design.

The method proposed in section 5.1 can not handle the interpretation of figure 15 . Indeed the functional matching can result in the following: $\{\mathrm{R} 2$ (ref), R1(impl), $\mathrm{R} 2$ (impl)\} are matched, and $\mathrm{R} 1\{$ ref) is unmatched as its input function contains don't care. Reintroducing the input function of FF R1(ref) to its output results in the following:

Out1ref $=\left(\mathrm{DC}^{\prime} \wedge\left(\neg \mathrm{V}^{\prime}\right)\right)$

Out1impl $=\mathrm{V} 1$

=>Out1impl $\not \subset$ Out1ref (false negative)

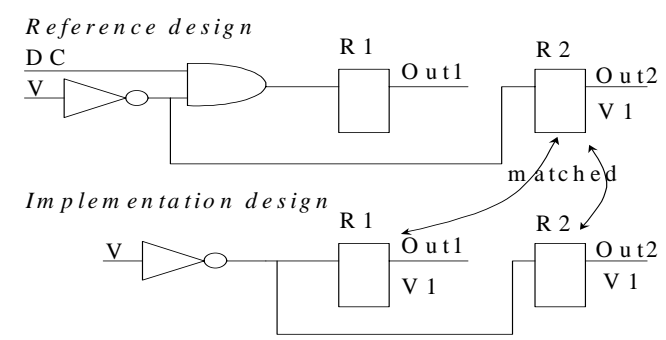

Figure 15: Unknown interpretation of don't care $(\mathrm{DC}=1)$

The method proposed in [2] which is based on "inclusion matching" can not handle the interpretation of figure 16 . Indeed, functional matching using "inclusion checking" can match $\{\mathrm{R} 1$ (impl), R2(ref), R2(impl)\} because $\mathrm{R} 2$ (impl) is included in R1(Ref). In this case the proof result is Out1(ref) $\neq$ Out 1 (impl) (false negative)

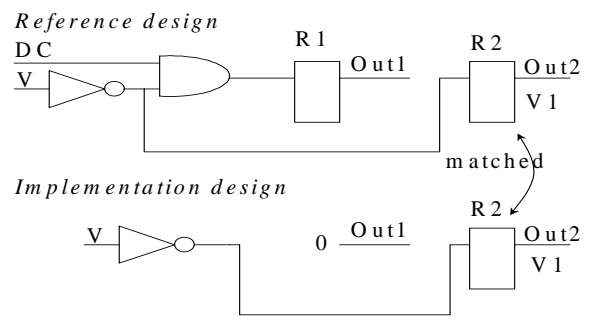

Figure 16: Unknown interpretation of don't care $(\mathrm{DC}=0)$

To handle 0-dc-func (1-dc-func), our method combines "inclusion matching" [2] with method presented in section 5.1 to extract assertions for prove of equivalence.

The algorithm is as follow:

1) Reintroduce the input function $F(t)$ of FFs with don't care variables to its output at next clock cycle.

2) Do the inclusion check.

3) Set all assertion given by inclusion checking (i.e if R is a matched $\mathrm{FF}$ with output variable $\mathrm{v}$ and it has been checked being included into FF R' with input function $\mathrm{F}(\mathrm{t})$, then the assertion $\mathrm{v} \subset \mathrm{F}(\mathrm{t})$ is set).
The algorithm is applied after functional matching which, is done without any consideration of don't cares.

Applying this method on Figure 15 gives:

1) Out1ref $=\left(\mathrm{DC}^{\prime} \wedge\left(\neg \mathrm{V}^{\prime}\right)\right)$

2) Inclusion check gives $\mathrm{R} 1(\mathrm{impl}) \subset \mathrm{R} 1$ (ref), R2(impl)

$\subset \mathrm{R} 1$ (ref)

3)Set the assertion $\mathrm{V} 1 \subset\left(\mathrm{DC}^{\prime} \wedge\left(\neg \mathrm{V}^{\prime}\right)\right)$

$\Rightarrow$ Out1impl $\subset$ Out1ref as we made the assertion that

V1 is contained in (DC' $\left.\wedge\left(\neg V^{\prime}\right)\right)$

Applying this method on Figure 16 gives:

1)Out1ref $=\left(\mathrm{DC}^{\prime} \wedge\left(\neg \mathrm{V}^{\prime}\right)\right)$

2)Inclusion check gives $\mathrm{R} 2$ (impl) $\subset \mathrm{R} 1$ (ref)

3) Set the assertion $\mathrm{V} 1 \subset\left(\mathrm{DC}^{\prime} \wedge\left(\neg \mathrm{V}^{\prime}\right)\right)$

$\Rightarrow$ Outlimpl $\subset$ Out1ref as we have 0 contained in function $\left(\mathrm{DC}^{\prime} \wedge\left(\neg \mathrm{V}^{\prime}\right)\right)$

\subsection{Improvements and Limitations}

The method presented in section 5.2 needs to reintroduce the input function of FFs with don't care variables to its output at next clock cycle. If the FFs have a feedback loop, the method cannot work because of the loop. In this case, methods to transform circuit with feedback loop into loop free circuit can be used as presented in [4]. But this method cannot handle all kind of circuits; this is why we are working to settle a general method in this case.

In this paper, a set of potential optimizations, which removed redundant FFs, have been presented. This set is not exhaustive and new algorithm can be added to our engine to handle more optimizations.

\section{Experimental results \& Conclusion}

We have synthesized a set of industrial designs using Synplify Pro 7.0 (www.synplicity.com) synthesis tool with "prune redundant FFs option" enabled. For constant input and non-observable FFs, both our method and those of industrial tools have the same results. However, for all designs with stuck at and constant FFs, our method is better and is able to complete the proof successfully compared to the industrial tools (the designs $\mathrm{T} 7$ which is snippet of the circuit on figure10 in section 4 is proved correct with our method and not with the industrial tools). For circuits with don't care function, our method is able to perform the proof in all cases. The industrial tools can prove some of them. Our belief is that it randomly assigns a value to the don't care variables, and thus, this assignment can chose the same value as the synthesis tools (see T10 Figure15 section 5). This is confirmed by the fact that design T11 (Figure16 section 5), a different interpretation of the don't care variables of the same reference design of $\mathrm{T} 10$, is proved different.

Finally, we also notice an increase in run-time with our method. This is due to the "inclusion checking," which is needed for each possible pair. Our future work is to 
use simulation to reduce the number of possible pairs and thus decrease the run-time.. (Note: DC case 1: dc-fullfunc; DC case 2: 0-dc-func or 1-dc-func, SAt0: stuck-at0 FF, NObs: Non observable FF)
Design Automation Conference Proceedings 1996, 33rd , 3-7 June 1996

\begin{tabular}{|l|l|l|l|l|l|l|}
\cline { 4 - 7 } \multicolumn{2}{c|}{} & \multicolumn{2}{l|}{$\begin{array}{l}\text { Industrial } \\
\text { tools }\end{array}$} & \multicolumn{2}{l|}{$\begin{array}{l}\text { 1] } \\
\text { method }\end{array}$} \\
\hline Test & Size(K) & Pruning type & Pass & T(s) & Pass & T (s) \\
\hline T1 & 18 & NObs & Yes & 1.10 & Yes & 1.32 \\
\hline T2 & 33 & SAt0 +const & No & 1.89 & Yes & 4.73 \\
\hline T3 & 40 & Const+NObs & Yes & 2.12 & Yes & 1.21 \\
\hline T4 & 36 & Const+NObs & Yes & 1.79 & Yes & 3.40 \\
\hline T5 & 15 & Const & Yes & 1.46 & Yes & 2.03 \\
\hline T6 & 45 & No red & Yes & 24.8 & Yes & 35.26 \\
\hline T7 & 90 & SAt0 +const & No & 57.7 & Yes & 78.68 \\
\hline T8 & 102 & DC case 1 & Yes & 12.3 & Yes & 15.59 \\
\hline T9 & 200 & $\begin{array}{l}\text { Const+ DC } \\
\text { case1 }\end{array}$ & No & 32.3 & Yes & 36.23 \\
\hline T10 & 200 & DC case 2 & Yes & 35.2 & Yes & 54.56 \\
\hline T11 & 200 & DC case 2 & No & 40.1 & Yes & 58.23 \\
\hline
\end{tabular}

Table 1: Results on industrial designs

\section{References}

[1] C. van Eijk, "Sequential equivalence checking without state traversal",DATE,98, pp. 618-623.

[2] Anastasakis, Damiano, Tony Ma, Stanion, "A Practical and Efficient Method for Compare-point Matching" DAC 2002, pp 305-310.

[3] J.R Burch and V. Singhal "Robust Latch Mapping for Combinational Equivalence checking" in ICCAD, 1998, pp563-569.

[4] Ranjan, Singhal, Somenzi, Brayton, "Using Combinational Verification for Sequential Circuits" (DATE '99) p. 138

[5] C.vanEijk: Formal Methods for the Verification of Digital Circuits, Ph.D. Thesis of the Eindhoven University of Technology, Eindhoven, The Netherlands, September 1997

[6] Shi-Yu Huang, Kwang-Ting Cheng, Kuang-chien Chen, Uwe Glaeser, "An ATPG-based Framework for Verifying Sequential Equivalence" IEEE ITC 1996, pp. $145-157$

[7] Stefan Hendriex, luc Claesen, "Formally Verified Redundancy Removal", DATE 99, p. 150

[8] R.K.Brayton "sequential equivalence checking" Logic synthesis and verification. Kluwer Academic Publishers. Chap12

[9] Jerry R. Burch, Vigyan Singhal "Tight integration of Combinational Verification Methods", ICCAD98, San Jose p570-576.

[10] O.Coudert, C.Berthet, J-C Madre, "Verification of synchronous Sequential Machines based on symbolic execution", in "Automatic Verification Methods For Finite State Systems" J.Sifakis, LNCS n407, pp3658373, Springer Verlag 89

[11] Iyer, M.A.; Long, D.E.; Abramovici, M.; "Identifying sequential redundancies without search" in 